\title{
Single Walled Carbon Nanotubes Induce Indirect Cytotoxicity by Medium Depletion in A549 Lung Cells
}

Gordon Chambers

Technological University Dublin, Gordon.chambers@tudublin.ie

Alan Casey

Technological University Dublin, alan.casey@tudublin.ie

Fiona Lyng

Technological University Dublin, fiona.lyng@tudublin.ie

See next page for additional authors

Follow this and additional works at: https://arrow.tudublin.ie/scschphyart

Part of the Biochemistry, Biophysics, and Structural Biology Commons

\section{Recommended Citation}

Casey, A., Herzog, E., Lyng, F. M., Byrne, H. J., Chambers, G. and Davoren, M. "Single walled carbon nanotubes induce indirect cytotoxicity by medium depletion in A549 lung cells." Toxicology Letters, 179, 78-84 (2008) doi:10.1016/j.toxlet.2008.04.006

This Article is brought to you for free and open access by the School of Physics \& Clinical \& Optometric Science at ARROW@TU Dublin. It has been accepted for inclusion in Articles by an authorized administrator of ARROW@TU Dublin. For more information, please contact arrow.admin@tudublin.ie, aisling.coyne@tudublin.ie, gerard.connolly@tudublin.ie.

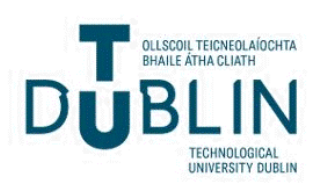




\section{Authors}

Gordon Chambers, Alan Casey, Fiona Lyng, Maria Davoren, Eva Herzog (Thesis), and Hugh Byrne

This article is available at ARROW@TU Dublin: https://arrow.tudublin.ie/scschphyart/25 


\title{
Single walled carbon nanotubes induce indirect cytotoxicity by mealum depletion in A549 lung cells
}

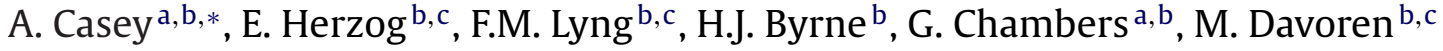 \\ a School of Physics, Dublin Institute of Technology, Kevin Street, Dublin 8, Ireland \\ ${ }^{\mathrm{b}}$ Focas Institute, Dublin Institute of Technology, Kevin Street, Dublin 8, Ireland \\ ${ }^{\mathrm{c}}$ Radiation and Environmental Science Centre (RESC), Focas Institute, Dublin Institute of Technology, Kevin Street, Dublin 8, Ireland
}

\section{A R T I C L E I N F O}

\section{Article history}

Received 1 February 2008

Received in revised form 9 April 2008

Accepted 11 April 2008

Available online 22 April 2008

\section{Keywords}

Carbon nanotubes

Cytotoxicity

Clonogenic

Depletion

\begin{abstract}
A B S T R A C T
The ability of two types of single walled carbon nanotubes (SWCNT), namely Arc Discharge (AD) a $\mathrm{HiPco}^{\circledR}$ single walled carbon nanotubes, to induce an indirect cytotoxicity in A549 lung cells by means u medium depletion was investigated. The nanotubes were dispersed in a commercial cell culture medium and subsequently removed by centrifugation and filtration. Spectroscopic analysis confirmed the removal of the nanotubes and showed differing degrees of alteration of the composition of the medium upon the removal of the nanotubes. The ability to induce an indirect cytotoxic effect by altering the medium was evaluated using two endpoints, namely the Alamar Blue (AB) and the Clonogenic assay. Exposure of the A549 cells to the depleted medium which had previously contained carbonaceous nanoparticles, revealed significant cytotoxicity for both endpoints employed. The results presented demonstrate that single walled carbon nanotubes can induce an indirect cytotoxicity by alteration of cell culture medium (in which they have previously been dispersed) which potentially results in a false positive toxic effect being observed in cytotoxicity studies.
\end{abstract}

\section{Introduction}

Single walled carbon nanotubes (SWCNT) interact strongly with a large range of molecular species from dyes to polysaccharides and bio-molecules (Bandyopadhyaya et al., 2002; Chambers et al., 2003; Casey et al., 2005; Dalton et al., 2000; Hedderman et al., 2004; Keogh et al., 2004; Moulton et al., 2005; Salvador-Morales et al., 2006). Spectroscopic analysis of a commercial cell culture medium, commonly used in toxicity studies, revealed significant interaction of the constituent components with SWCNT. It was shown in an earlier study (Casey et al., 2007a) that on dispersal of SWCNT in the cell culture medium significant colour changes were observed in the medium. These changes indicated a degree of molecular interaction between the SWCNT and the constituents of the medium. Spectroscopic analysis confirmed the presence of these interactions with a loss of the associated absorption and fluorescent emission of the medium components. The question was thus raised that if the SWCNT interact with the medium and adsorb nutrient constituents, does the medium still have the same capability to maintain healthy cells? Furthermore, can the toxicological

\footnotetext{
* Corresponding author. Tel.: +3531 4027932; fax: +35314027901.

E-mail address: alan.casey@dit.ie (A. Casey).
}

data collected on experiments performed in this way (dispersion of SWCNT in a cell culture medium) be affected? So it is postulated that SWCNT may induce an indirect toxicity due to the reduction in the availability of the medium components to the cells due to interaction with the SWCNT. This study will investigate this hypothesis of an indirect toxicity by medium depletion. In vitro cytotoxicity studies were thus carried out on A549 lung cells with two types of carbon nanotubes for comparative purposes, namely $\mathrm{HiPco}^{\circledR}$ SWCNT and Arc Discharge (AD) SWCNT. Various concentrations of the carbon-based nanoparticles were dispersed in cell culture medium and then removed by a process of centrifugation and filtration. Healthy confluent cells were then exposed to this filtered medium and cellular viability was estimated using two cytotoxicity endpoints, namely the Alamar Blue (AB) and the clonogenic assays. Spectroscopic analysis was performed on all test media samples firstly to verify the complete removal of the nanoparticle in question from the suspension and secondly to investigate the effect of the removal of the nanoparticles on the constituents of the medium itself.

\section{Materials and methods}

2.1. Test materials and reagents

HiPco ${ }^{\circledR}$ SWCNT were purchased from Carbon Nanotechnologies, Inc. (Houston, TX). This material contained up to $10 \mathrm{wt} \%$ residual Fe catalyst particles. Arc Discharge 
SWCNT, containing transition metal impurities namely Ni (3wt\%) and Yt ( $2 \mathrm{wt} \%$ ) and amorphous carbon were purchased from Sigma Aldrich Ltd. (Dublin, Ireland). Alamar Blue ${ }^{\mathrm{TM}}$, cell culture media and supplements and the trypsinisation solution were all purchased from Biosciences (Dublin, Ireland).

\subsection{Preparation of test samples}

The solutions were prepared by dispersing an initial concentration of $0.8 \mathrm{mg} / \mathrm{ml}$ of both types of SWCNT in cell culture medium supplemented with $5 \%$ foetal bovine serum using an ultrasonic tip. Each stock concentration was then serially diluted with culture medium and sonicated as before to prepare test concentrations. As previously reported (Casey et al., 2007a), the addition of the serum had an effect on the dispersion behaviour of the SWCNT and the recommendation of working in the absence of serum in toxicity studies was made. In this current study to facilitate the investigation of longer incubations (e.g. 72 and $96 \mathrm{~h}$ exposure tests), test concentrations were prepared in $5 \%$ serum media to facilitate the healthy growth of cells over this extended time period. After dispersion, the test concentrations were refrigerated for $24 \mathrm{~h}$ and then underwent a process of centrifugation (3000RPM/1800G for $20 \mathrm{~min}$ ) and filtering (using $0.2 \mu \mathrm{m}$ cellulose acetate filters) to remove the dispersed nanoparticles. This resultant filtrate was termed depleted medium. Cells were then exposed to the depleted medium that was previously exposed to a concentration range of nanoparticles $(0.00156-0.8 \mathrm{mg} / \mathrm{ml})$.

\subsection{Characterisation}

For cytotoxicity evaluation, fluorescence and absorbance were all quantified using a microplate reader (TECAN GENios, Grödig, Austria). Absorption and fluorescence spectroscopy were performed on the dispersions of nanotubes in all solutions after a $24 \mathrm{~h}$ settling period after which they were characterised using a PerkinElmer Lambda 900 Absorption and LS55B Luminescence spectrometers, respectively. Raman analysis was performed using an Instruments S.A. LabRam 1B Raman microscope using $514.5 \mathrm{~nm}$ laser excitation on drop cast samples. Elemental analysis was performed by Atomic Absorption (AA) spectroscopy on media suspensions post-SWCNT removal with the aid of a Varian SpectrAA 200.

\subsection{Cell culture}

The human alveolar carcinoma epithelial cell line A549 (ATCC, CCL-185) was employed for toxicity evaluation. Cells were grown in F-12K medium (Kaighn's Modification, Gibco) supplemented with $10 \%$ foetal calf serum (FCS), $45 \mathrm{IU} \mathrm{ml}^{-1}$ penicillin and $45 \mu \mathrm{g} \mathrm{ml}^{-1}$ streptomycin and grown in a humidified incubator at $37^{\circ} \mathrm{C}\left(5 \% \mathrm{CO}_{2}\right)$.

\subsection{Cytotoxicity evaluation}

For the AB assay, cells were seeded at a density of $1 \times 10^{5}$ cells $/ \mathrm{ml}$ for the $24 \mathrm{~h}$ test $7 \times 10^{4}$ cells $/ \mathrm{ml}$ for the $48 \mathrm{~h}$ test, $3 \times 10^{4}$ cells $/ \mathrm{ml}$ for the $72 \mathrm{~h}$ test and $2 \times 10^{4}$ cells $/ \mathrm{ml}$ for the $96 \mathrm{~h}$ test in 96 -well plates (Nunc, Denmark). These seeding densities were found to be optimal to achieve the desired confluency for each cell line at the end of each respective exposure period. Cells were allowed to attach for $24 \mathrm{~h}$ before they were washed with $100 \mu$ l of phosphate buffered saline (PBS) and treated with $100 \mu \mathrm{l}$ depleted medium (previously containing test nanoparticle concentrations of $0.00156-0.8 \mathrm{mg} / \mathrm{ml}$ ). Upon exposure for $24,48,72$ or $96 \mathrm{~h}$, cell viability was assessed using the $\mathrm{AB}$ assay according to the manufacturer's guidelines. At least three independent experiments were performed.

The procedure for the clonogenic assay was adapted from Puck and Markus (1956) and Franken et al. (2006) and cells were treated after plating. Exponentially growing cells were harvested and seeded in 6-well microplates (Nunc, Denmark) at a density of 250 cells/well. Each well-contained $2 \mathrm{ml}$ of cell culture medium. Cells were allowed to attach for approximately $14 \mathrm{~h}$. This attachment period was shorter than the population doubling time of these cell lines which is reported to be around $22 \mathrm{~h}$ for A549 cells (ATCC, CCL-185) so that single cells were present at the start of exposure. Cells were then washed with $2 \mathrm{ml}$ of PBS and treated with $2 \mathrm{ml}$ depleted medium. Following exposure to the depleted medium for 10 days, cells were washed with PBS and finally fixed and stained using a $20 \%$ carbol fuchsin in formalin solution (BDH, Poole, UK) and the number of cell colonies was determined. In addition to colony number as the classical clonogenic assay endpoint, surface area was chosen as an additional endpoint to evaluate the effects of the depleted medium on colony formation (Spadinger et al., 1994). Digital photographs were taken from each 6-well microplate and the Java based image-processing program ImageJ (http://rsb.info.nih.gov/ij/) was used to measure colony surface areas.

\subsection{Statistics}

At least three independent experiments were conducted for each type of nanoparticle and toxicity endpoint. Test results for each assay were expressed as percentage of the unexposed control \pm standard deviation (S.D.). Control values were set as $100 \%$. Differences between samples and the control were evaluated using the statistical analysis package SPSS 14.0. Statistically significant differences were set at $p \leq 0.05$. Normality of data was confirmed with $\mathrm{Q}-\mathrm{Q}$ percentile plots and Kolmogorov-Smirnov tests. Equality of variances was evaluated using Levène tests. One-way analysis of variances (ANOVA) followed by Dunnett's multiple comparison tests were carried out for normally distributed samples with homogeneous variances. Non-parametric tests, namely Kruskal-Wallis followed by Mann-Whitney- $U$-tests were applied to samples without normal distribution and/or inhomogeneous variances.

\section{Results and discussion}

In an earlier study it was observed that upon the dispersion of the SWCNT within the medium, there was considerable interaction between the SWCNT and the various components of the cell culture medium with a resultant colour change in the higher concentration regions $(0.4-0.8 \mathrm{mg} / \mathrm{ml}$ ) (Casey et al., 2007a). It should be noted that the interactions were observed irrespective of the presence or absence of the serum. In the aforementioned study it was postulated that these interactions may reduce the availability of the medium components to the cultured cells and may result in an indirect cytotoxicity due to medium depletion by interaction. This colour change was observed again here in all test samples (HiPco ${ }^{\circledR}$ SWCNT and AD SWCNT).

\subsection{Spectroscopic analysis}

In order to investigate the proposed mechanism of an indirect rather than a direct toxic effect of SWCNT, it is vital that the removal of the nanotube species in question be carried out. For the purpose of this study Raman spectroscopy was employed to verify the removal of nanotube from the medium after centrifugation and filtration. Raman analysis was carried out on all samples at $514.5 \mathrm{~nm}$ laser excitation. The Raman spectrum of pristine nanotubes consists primarily of three main features, the radial breathing mode (RBM), the D line and the G line (Brown et al., 2001; Dresselhaus et al., 2002; Jorio et al., 2001, 2002; Kukovecz et al., 2002; Kuzmany et al., 2001; Pimenta et al., 2001; Yu and Brus, 2001). The dominant feature in the Raman signal of SWCNT is the G line situated between 1450 and $1650 \mathrm{~cm}^{-1}$ the absence of this feature from the recorded spectra would indicate that the SWCNT were successfully removed from the medium.

Fig. 1 displays the recorded spectra for unfiltered medium containing HiPco SWCNT (Fig. 1a and b), and for all filtered media, HiPco $^{\circledR}$ SWCNT (Fig. 1d) and AD SWCNT (Fig. 1c). The pre-filtration spectra of $\mathrm{HiPco}^{\circledR}$ SWCNT treated media suspensions (Fig. 1a and b) were recorded from drop cast slides. In these spectra the dominant

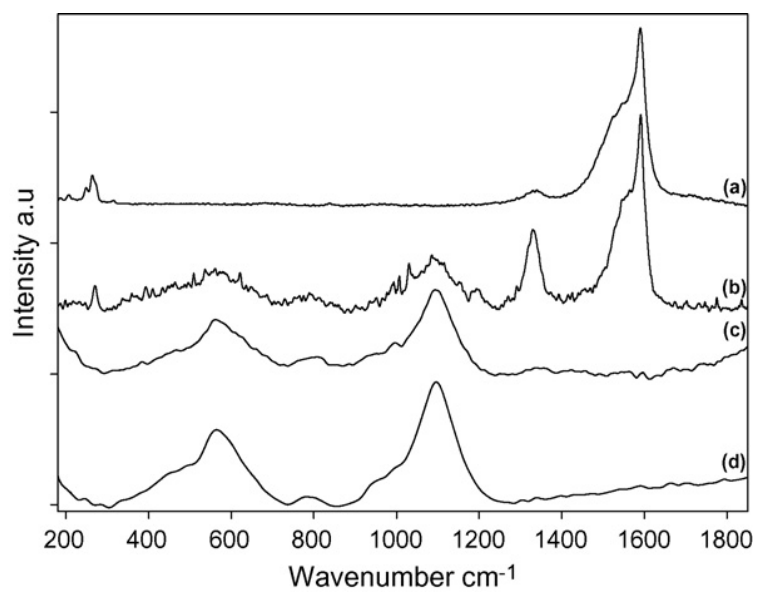

Fig. 1. Raman Spectra by $514.5 \mathrm{~nm}$ excitation of (a) unfiltered 5\% FBS medium containing HiPco SWCNT $0.8 \mathrm{mg} / \mathrm{ml}$, (b) Unfiltered 5\% FBS medium containing HiPco SWCNT $0.00156 \mathrm{mg} / \mathrm{ml}$, (c) Arc Discharge SWCNT filtrate $0.8 \mathrm{mg} / \mathrm{ml}$ and (d) HiPco SWCNT filtrate $0.8 \mathrm{mg} / \mathrm{ml}$. 
nanotube features can be clearly seen at both tested concentrations (0.8 (a) and 0.00156 (b) SWCNT $\mathrm{mg} / \mathrm{ml}$ ). Close examination of these spectra, in the RBM region, shows that there was minimal alteration to the aggregative state of the SWCNT. This correlates well with the results presented in an earlier study (Casey et al., 2007a) verifying that the tested SWCNT remained as bundles in all concentrations tested. Furthermore the difference in concentrations of these two samples gives a good representation of the fine quantities of SWCNT that can be detected spectrally by the Raman method. The absence of these features in the filtered samples would therefore provide a good indication of their removal by centrifugation and filtration. Fig. 1(c and d) displays the Raman spectra obtained by $514.5 \mathrm{~nm}$ excitation of the filtered samples ( $\mathrm{HiPco}^{\circledR}$ and AD SWCNT) of the highest test concentrations $(0.8 \mathrm{mg} / \mathrm{ml})$ recorded from drop cast slides. As can be seen, there is a complete absence of SWCNT features from the spectra obtained. This is a strong indication that the nanotubes have been essentially removed from the medium by centrifugation and filtration allowing the proposed hypothesis of an indirect toxicity by medium depletion to be investigated. The Raman analysis also shows no evidence of amorphous carbon (present as an impurity in AD SWCNT) although it is of course plausible that trace amounts remained within the media and was not detected by the Raman method. The HiPco SWCNT tested contain an impurity of remnant catalytic iron particles (10 wt\%), therefore, in order for any cytotoxicity observed to be attributed to that of an indirect effect it was vital that the associated iron impurities were removed with the SWCNT during filtration and centrifugation. Prior to toxicity testing, Atomic Absorption analysis was carried out to monitor iron levels within the media pre- and post-SWCNT removal. In $5 \%$ FBS medium an initial iron level of $1.98 \times 10^{-5} \mathrm{mg} / \mathrm{ml}$ was noted and after the addition and subsequent removal of the HiPco this value had increased slightly, in only the highest test concentration of $0.8 \mathrm{mg} / \mathrm{ml}$, by $1.02 \times 10^{-5}$ to $3 \times 10^{-5} \mathrm{mg} / \mathrm{ml}$. While a slight increase in iron levels were observed in the media following the filtration and centrifugation of the SWCNT, it should be noted that iron is an essential element for organisms and the levels present in the depleted medium were found to be two orders of magnitude less than those demonstrated to elicit genotoxic and cytotoxic effects in human cells (Lima et al., 2008).

The UV/visible absorption spectrum of 5\%-FBS-F12K medium, which consists of four features at 270, 360, 410 and $560 \mathrm{~nm}$ (Casey et al., 2007a), was seen to undergo considerable changes upon the addition of SWCNT. The features at 270 and $410 \mathrm{~nm}$ were assigned to the added 5\% FBS, whereas the two remaining features at 360 and $560 \mathrm{~nm}$ originate from the components of the medium itself. The feature at $360 \mathrm{~nm}$ can be attributed to riboflavin (Posadaz et al., 2000; Zirak et al., 2005), a vitamin present in the medium whereas the feature at $560 \mathrm{~nm}$ can be assigned to the phenol red indicator. The absorption spectrum of SWCNT in water suspensions consists of three broad absorption features centred approximately at 1200,800 and $270 \mathrm{~nm}$. The peaks at 1200 and $800 \mathrm{~nm}$ are highly structured and are attributed to optical transitions between mirror image spikes in the diameter dependent density of states, with the feature at $270 \mathrm{~nm}$ attributed to the $\pi$-plasma frequency of $\sim 4.6 \mathrm{eV}$ in $\pi$-conjugated carbon materials (Kataura et al., 1999). In all test solutions, a reduction in the phenol red peak at $560 \mathrm{~nm}$ was observed (Fig. 2a and b) which was as expected due to the colour change observed in the high SWCNT concentration region. However, only slight alterations in $\mathrm{pH}$ were registered with the $\mathrm{pH}$ decreasing for a value of 8.58 at the highest test concentration of $0.8 \mathrm{mg} / \mathrm{ml}$ to a value of 8.41 at the lowest test concentration of $0.00156 \mathrm{mg} / \mathrm{ml}$. This was interpreted as evidence that the SWCNT altered the chemical "composition" of the medium by interaction without adversely affecting the $\mathrm{pH}$. Further reductions of the features attributed to the added FBS supplement were observed, at 410

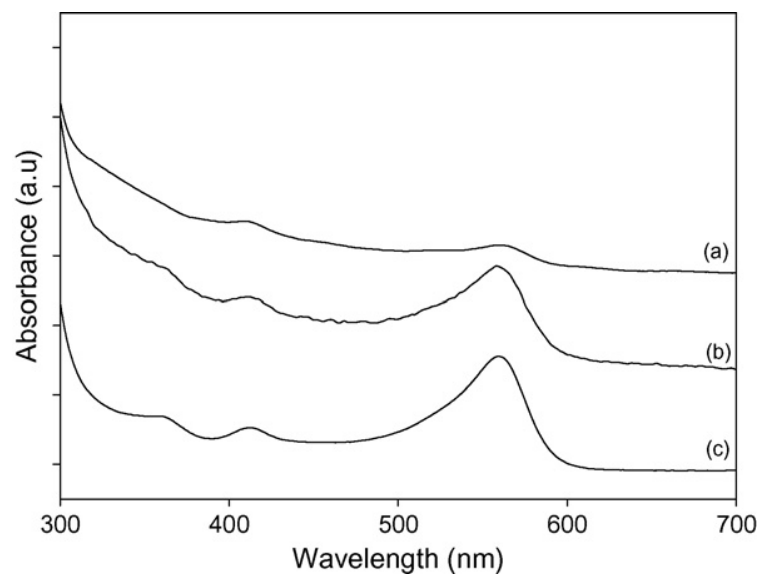

Fig. 2. UV-vis absorption spectra of (a) HiPco SWCNT Filtered medium $0.8 \mathrm{mg} / \mathrm{ml}$ (b) Arc Discharge SWCNT Filtered medium $0.8 \mathrm{mg} / \mathrm{ml}$ and (c) 5\% FBS medium.

and $270 \mathrm{~nm}$, in all tested filtered samples (Fig. 2a and b). The feature at $\sim 360 \mathrm{~nm}$, attributed to the vitamin riboflavin, was reduced in all tested filtered samples (Fig. 2a and b). The spectral changes observed here are attributable to an adsorption of the molecular components of the medium to the $\pi$-electron surface of the nanotubes. This adsorption coupled with the filtration and centrifuging would in turn cause a reduced effective concentration of these components in the medium facilitating the indirect toxic effect of SWCNT due to medium depletion to occur.

As described earlier reductions in absorbance were observed in the characteristic medium features so it was expected that there would be a reduction in their associated fluorescence emission features. Excitation of 5\% FBS medium by three wavelengths, namely 268, 360 and $410 \mathrm{~nm}$, each corresponding to the absorbance of an individual component of the medium, yielded emission spectra centred at 360, 450 and $450 \mathrm{~nm}$, respectively. These excitation wavelengths were used here to study the filtered medium to assess the effect of SWCNT removal. In all tested samples the emission, by excitation with the aforementioned wavelengths, was reduced. The quenching of emission was monitored as a function of initial particle concentration, and plotted as a ratio (Fig. 3a and b). Fluorescence studies have been shown to aid in the elucidation of the interaction of nanotubes with different molecular species (Keogh et al., 2005; Hedderman et al., 2006). In an earlier study (Casey et al., 2007a) a model derived by Coleman et al. (2004) was adapted and employed to assess the degree of interaction between SWCNT and cell culture medium. Eq. (1) represents the dynamic equilibrium at which the adsorption rate equals the desorption rate for fluorescent molecules in composite systems, where $N_{\mathrm{F}}$ is the number of free molecules, $N_{\mathrm{B}}$ is the number of bound molecules, $\mathrm{Fl}_{\text {comp }}$ is the fluorescence of the composite and $\mathrm{Fl}_{\text {molecule }}$ is the fluorescence of the pristine solution which gives the fraction of free molecules in solution. The model was derived for $1: 1$ ratios by mass and so for all concentrations the partial SWCNT concentration, $C_{\mathrm{NT}}$, equals the partial molecular concentration, $C_{\mathrm{m}}$.

$\frac{N_{\mathrm{F}}}{N_{\mathrm{F}}+N_{\mathrm{B}}}=\frac{1}{1+C_{\mathrm{NT}} / C_{0}}=\frac{\mathrm{Fl}_{\text {comp }}}{\mathrm{Fl}_{\text {molecule }}}$

$C_{0}$ is a characteristic concentration associated with the interaction and is described by

$C_{0}=\frac{\pi^{2} v \rho_{\text {Bun }} A_{\text {Bun }} \mathrm{e}^{-E_{\mathrm{b}} / k T}}{48 \mathrm{Df}}$

$C_{0}$ may be considered as a ratio of the desorption rate to the adsorption rate, for the purpose of this study this model will be employed assess the degree of interaction or alteration of the cell culture 

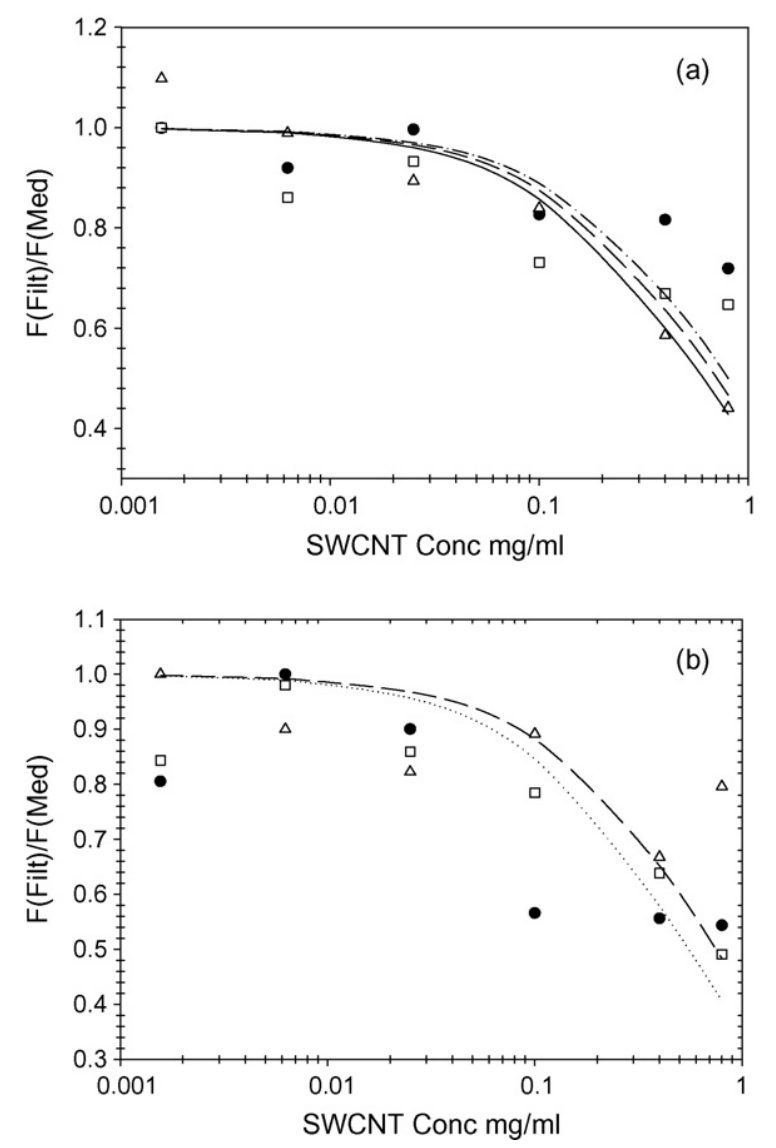

Fig. 3. Plot of emission ratios of $5 \%-F B S-F 12 K$ filtered cell culture medium against (a) HiPco SWCNT and (b) Arc Discharge SWCNT concentration (initial) by excitation at $(\square) 268 \mathrm{~nm}$ excitation, $(\bullet) 360 \mathrm{~nm}$ excitation and $(\triangle) 410 \mathrm{~nm}$ excitation.

medium upon the dispersal and subsequent removal of SWCNT within the medium. This model facilitates characteristic concentrations $\left(C_{0}\right)$ to be estimated for the molecule under test. This $C_{0}$ value was then used to estimate the degree of interaction between the SWCNT and the relevant molecule. According to Eq. (2) the $C_{0}$ value is inversely proportional to the binding energy $\left(E_{\mathrm{b}}\right)$ the nature of this relationship would imply that a low $C_{0}$ value would correspond to a higher $E_{\mathrm{b}}$ (higher degree of interaction) and similarly a high $C_{0}$ value would correspond to a lower $E_{\mathrm{b}}$ hence a lesser degree of interaction.

As can be seen in Fig. 3 the emission ratio plots follow an approximate trend to that proposed by the original model (Coleman et al., 2004). However, a significant degree of deviation from the model fit can be observed. This increased degree of deviation can be attributed to the increased variability introduced to the system by the filtration and centrifugation process. Despite the inability to reliably fit the data to the model proposed by Coleman et al. (2004) it can be clearly seen in the ratio plots of Fig. 3 that the incorporation and removal of the carbon based nanoparticles into the medium altered its emission characteristics giving further supportive evidence for the alteration of the medium upon interaction with the SWCNT. Although a similar fit is observed for the medium exposed to both types of SWCNT tested, it should be noted that a maximum degree of quenching was observed for all emission features at the highest concentrations of HiPco tubes $(0.8 \mathrm{mg} / \mathrm{ml})$. The relative equivalence of the responses also indicates that the impurities play a negligible role in the medium depletion, given the differing nature of the impurities in the two samples. As reported previously, bundles of HiPco SWCNT present a larger surface area when compared to that of arc discharge SWCNT (Hedderman et al., 2006; Herzog et al., 2007). A larger surface area would in turn lead to increased nutrient adsorption onto HiPco SWCNT bundles compared to arc discharge SWCNT bundles. This would lead to a more effective depletion of culture medium by HiPco SWCNT. These reductions in emission, coupled with the reductions in the associated absorbance of medium features, provide further evidence of a reduction in the effective concentrations of these medium components facilitating an indirect cytotoxicity to be induced by means of interaction with the SWCNT.

\subsection{Cytotoxicity evaluation}

Due to the reported interaction between organic indicator dyes and SWCNT, employing such dyes for cytotoxicity screening of carbon nanoparticles is not recommended (Hurt et al., 2006; Monteiro-Riviere and Inman, 2006; Wörle-Knirsch et al., 2006; Casey et al., 2007a,b). However, the absence of SWCNT in the tested medium made it possible to employ the highly sensitive Alamar Blue assay. In contrast to other routinely used indicator dyes (e.g. the MTT and neutral red assays) the AB dye has the advantage of being water soluble, stable in the culture medium and nontoxic, so that continuous monitoring of cell cultures is permitted, and viability of cells is not altered. Figs. 4 and 5 display the cytotoxicity response curves obtained for $\mathrm{HiPco}^{\circledR}$ SWCNT and the arc discharge SWCNT respectively using the AB assay following exposure to the filtered medium. Again concentrations quoted are those of the nanoparticles in the medium pre-filtration. Fig. 4 displays the cytotoxicity response curve obtained for filtered medium which previously contained HiPco SWCNT. As can be seen, there is a clear dose- and time-dependent decrease in cell viability starting at SWCNT concentrations of $0.4 \mathrm{mg} / \mathrm{ml}$ after $48 \mathrm{~h}$ exposure. However, the effect only fell within statistical significance at the highest test concentration of $0.8 \mathrm{mg} / \mathrm{ml}$.

A slightly different behaviour was observed for the arc discharge SWCNT filtered medium (Fig. 5). Data variability was larger than seen for HiPco SWCNT filtered medium and a clear dose- and timedependent effect could only be noted at the highest concentrations tested. However, it is clear for example from the $96 \mathrm{~h}$ response that the arc discharge exposed medium elicits a lesser response than that for the corresponding HiPco medium irrespective of statistical significance. This is consistent with the greater degree of quenching observed for the HiPco medium in Fig. 3 above. Again the greater surface area reported for HiPco bundles compared to those of arc discharge tubes could explain the higher toxicity observed for medium previously exposed to HiPco SWCNT than arc discharge SWCNT filtered medium.

In addition to the $A B$ assay, the clonogenic assay, an alternative assay not including any absorbance or fluorescence measurements of indicator dyes was performed (Herzog et al., 2007). The clonogenic assay is an in vitro cell survival based assay measures the ability of a single cell to form a colony (Puck and Markus, 1956). Only mitotically viable cells are able to produce progenitor cells, therefore the number of colonies formed following or during treatment is an excellent indicator for cell viability. Using colony size measurements as an additional endpoint can give an estimation of the rate of division and proliferation of treated cells (Horáková et al., 2001 ). The clonogenic assay has been reported to be more sensitive than colourimetric cytotoxicity assays since dying, abortive cells can still be metabolically active and thus give misleading results in non-clonogenic assays (Rober and Drewinko, 1976). It has the advantage of allowing the distinction between effects on cell viability and cell proliferation, can show long-term effects on a sub-lethal level and has recently been shown to be suitable for the assessment of carbon nanoparticle toxicity (Herzog et al., 2007). Results of A549 


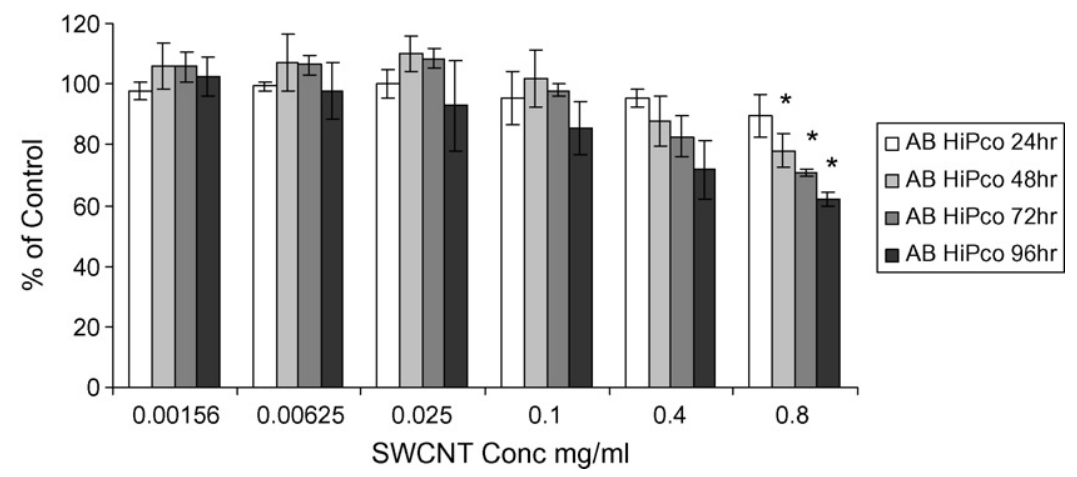

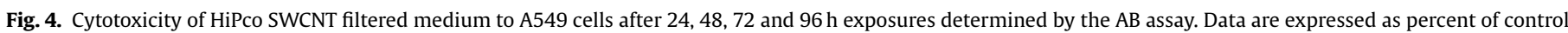
mean \pm S.D. of four independent experiments. $\left({ }^{*}\right)$ Denotes significant difference from control $(p \leq 0.05)$.

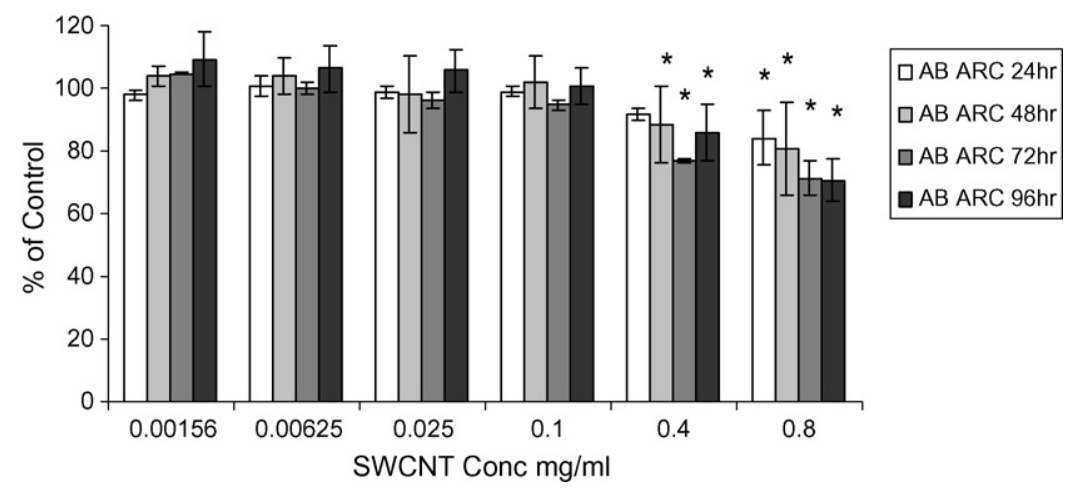

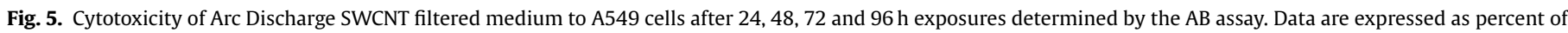
control mean \pm S.D. of three independent experiments. $\left({ }^{*}\right)$ Denotes significant difference from control $(p \leq 0.05)$.

lung cell exposure to culture medium previously exposed to SWCNT are shown in Figs. 6 and 7.

As can be seen in Fig. 7, the ability of A549 cells to form colonies during incubation in depleted culture medium was only slightly reduced when the medium was previously exposed to the highest SWCNT concentration of $0.4 \mathrm{mg} / \mathrm{ml}$. Treatment with any other SWCNT concentrations did not show any effects. This was the same for HiPco and arc discharge SWCNT samples. However, when determining colony sizes, significant, dose-dependent reductions could be observed following HiPco ${ }^{\circledR}$ SWCNT treatment where colonies reached sizes of only $51 \%$ of that of controls grown in normal culture medium. Already at the lowest $\mathrm{HiPco}^{\circledR}$ SWCNT concentration tested, colony size was reduced by more than $26 \%$. It is well known that cells in nutrient deficient environments respond by reducing their cell proliferation, ultimately leading to reduced colony sizes (Ozturk et al., 2003; Zenin et al., 1982).

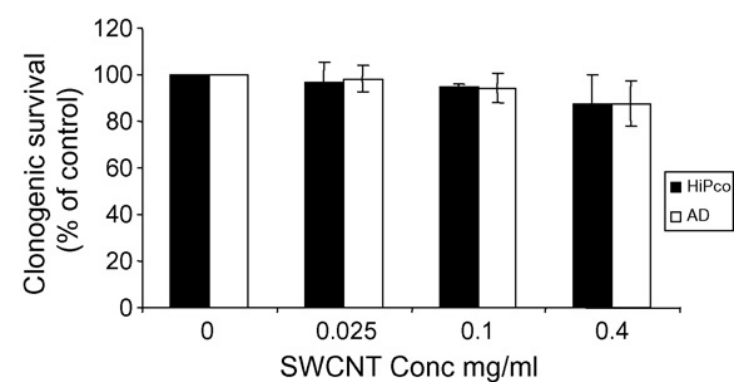

Fig. 6. Colony numbers of A549 cells following 10 days exposure to SWCNT depleted culture medium. Data are expressed as percent of control mean \pm S.D. of three independent experiments. $\left({ }^{*}\right)$ Denotes significant difference from control $(p \leq 0.05)$.
Our previous study demonstrated that colony size was a more sensitive endpoint than colony numbers following exposure of A549 cells to carbon nanoparticles including carbon black, $\mathrm{HiPco}^{\circledR}$ SWCNT and AD SWCNT samples (Herzog et al., 2007). The clonogenic results lend further support to the possibility of an indirect toxicity by means of nutrient depletion resulting from SWCNT interaction with the culture medium. SWCNT have been reported to interact and bind to a variety of organic molecules including sugars, proteins and culture medium components (Chambers et al., 2003; Casey et al., 2005, 2007a; Moulton et al., 2005). Monteiro-Riviere and Inman (2006) reported binding of the pro-inflammatory cytokine IL-8 to nanoparticle carbon black. Salvador-Morales et al. (2006) have demonstrated the binding of lung surfactant proteins onto SWCNT. This adsorptive nature of SWCNT may potentially lead to an indirect toxicity due to the binding and therefore removal of

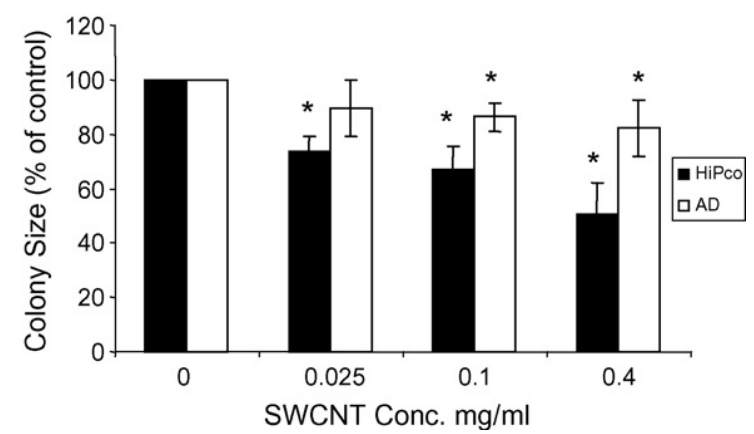

Fig. 7. Colony sizes of A549 cells following 10 days exposure to SWCNT depleted culture medium. Data are expressed as percent of control mean \pm S.D. of three independent experiments. $\left({ }^{*}\right)$ Denotes significant difference from control $(p \leq 0.05)$. 


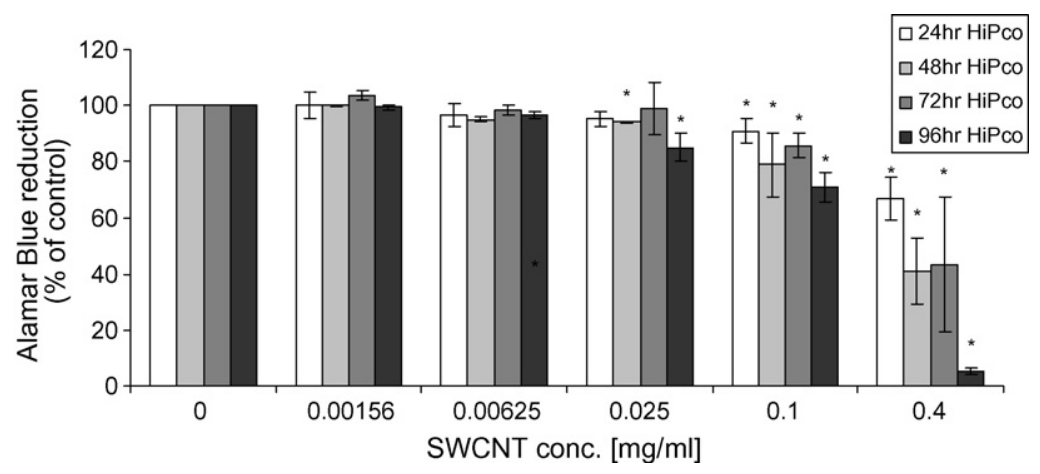

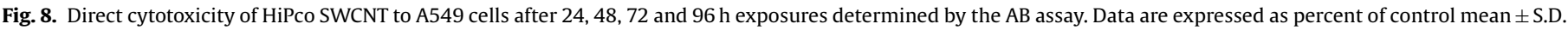
of three independent experiments. $\left({ }^{*}\right)$ Denotes significant difference from control $(p \leq 0.05)$.

essential nutrients, cytokines and/or growth factors. In this study it could be shown that SWCNT can lead to decreased cell viability due to such indirect mechanisms causing depletion of cell culture media. Medium depletion caused reduced metabolic activity, as seen by the AB assay, as well as inhibited colony formation as shown by the clonogenic (colony forming) assay. The dominant effect, as indicated by the colony size endpoint, is a reduction of the proliferative capacity of the cells in the depleted medium. During the clonogenic assay, on average $8 \mu$ l of culture medium was available for one cell during the 10 days of exposure. In contrast, during the AB assay, only $0.002,0.003,0.007$ and $0.01 \mu \mathrm{l}$ was available for 24 , 48,72 and 96 h exposures, respectively. Therefore, the total of available nutrients was higher during the clonogenic assay compared to the $A B$ assay. This could explain why cells were still able to form colonies at numbers comparable to cells grown in control medium. However, medium depletion did inhibit the rate of cell proliferation and metabolism as indicated by reduced Alamar Blue reductions and smaller colony sizes. As the amount of nutrients available to cells during the $\mathrm{AB}$ assay was $99.9 \%$ lower compared to the clonogenic assay, greater effects of medium depletion could be seen in the $\mathrm{AB}$ assay which can explain the discrepancies seen between the two assay systems employed in this study. The clonogenic assay, in contrast, could show that medium depletion has long-term effects on A549 cells on a sub-lethal level. HiPco SWCNT samples were always more effective compared to arc discharge SWCNT samples (Herzog et al., 2007) indicating the importance of surface area in the mechanism of medium depletion.

This data presented in this study has implications for direct exposure SWCNT cytotoxicity studies; Fig. 8 displays the obtained cytotoxic response curve of A549 cells following direct exposure to SWCNT as evaluated by the AB assay. Data of this nature would obviously contain a contribution from the demonstrated indirect effect of medium depletion coupled with absorptive interferences (Casey et al., 2007b; Hurt et al., 2006; Monteiro-Riviere and Inman, 2006; Wörle-Knirsch et al., 2006) from remnant SWCNT after initial exposure both contributing to false positive toxic effects being recorded when evaluating high concentration SWCNT exposures.

\section{Conclusions}

This study has used spectroscopic analysis coupled with cytotoxicity assessment to verify the proposed hypothesis of an indirect toxicity as a result of the interaction of SWCNT with cell culture medium. To confirm this effect, spectroscopic analysis was performed to verify the removal of the SWCNT and associated impurities from the medium by centrifugation and filtration. Raman spectroscopy gave no indication of the presence of SWCNT in the filtered samples suggesting they were sufficiently removed during the test sample preparation process. It is without question that upon their dispersion and subsequent removal, both types of SWCNT tested in this study did alter the composition of the medium, as evidenced by the spectroscopic analysis. The evident removal of impurities highlights that this is an intrinsic effect of the adsorption by the nanotubes of molecular components of the cell growth medium resulting in medium depletion. This in turn resulted in an adverse effect on cellular growth as verified by the cytotoxicity data presented for the $\mathrm{AB}$ and the clonogenic assays. The clonogenic endpoints give a clear indication that the principal effect is one of reduced proliferative capacity rather than viability. Once again cellular effects were higher for the nanoparticle with the larger surface area. As these media interactions and absorptive interferences with carbon nanomaterials have only come to light in recent years (Hurt et al., 2006; Monteiro-Riviere and Inman, 2006; Wörle-Knirsch et al., 2006; Casey et al., 2007a,b), there is the risk that toxicity observations to date have contained a contribution from this indirect effect, i.e. a false positive result rather than the inherent toxicity of the nanomaterial. Care is therefore warranted when interpreting toxicity data generated with carbonaceous nanoparticles. This study further highlights the importance of assessing all contributing factors, such as the discussed indirect cytotoxicity effects when preparing a toxicity profile for these types of nanoparticles in vitro.

\section{References}

Bandyopadhyaya, R., Nativ-Roth, E., Regev, O., Yerushalmi-Rozen, R., 2002. Stabilization of individual carbon nanotubes in aqueous solutions. Nanoletters 2, 25-28.

Brown, S.D.M., Jorio, A., Dresselhaus, M.S., Desselhaus, G., 2001. Observations of the $\mathrm{D}$-band feature in the Raman spectra of carbon nanotubes. Physical Review B 64, 073403-1-073403-4.

Casey, A., Farrell, G.F., McNamara, M., Byrne, H.J., Chambers, G., 2005. Interaction of carbon nanotubes with sugar complexes. Synthetic Metals 153, 357-360.

Casey, A., Davoren, M., Herzog, E., Lyng, F.M., Byrne, H.J., Chambers, G., 2007a. Probing the interaction of single walled carbon nanotubes within cell culture medium as a precursor to toxicity testing. Carbon 45, 34-40.

Casey, A., Herzog, E., Davoren, M., Lyng, F.M., Byrne, H.J., Chambers, G., 2007b. Spectroscopic analysis confirms the interactions between single walled carbon nanotubes and various dyes commonly used to assess cytotoxicity. Carbon 45 , 1425-1432.

Chambers, G., Carroll, C., Farrell, G.F., Dalton, A.B., McNamara, M., in het Panhuis, M., Byrne, H.J., 2003. Characterization of the interaction of gamma cyclodextrins with single walled carbon nanotubes. Nanoletters 3 (6), 843-846.

Coleman, J.N., Maier, S., Fleming, A., O'Flaherty, S., Minett, A., Ferreira, M.S., Hutzler, S., Blau, W.J., 2004. Binding kinetics and spontaneous single wall carbon nanotube bundle dissociation in low concentration polymer-nanotube solution. Journal of Physical Chemistry B 108, 3446-3450.

Dalton, A.B., Stephan, C., Coleman, J.N., McCarthy, B., Ajayan, P.M., Lefrant, S., Bernier, P., Blau, W.J., Byrne, H.J., 2000. Selective interaction of a semi conjugated organic polymer with single-wall nanotubes. Journal of Physical Chemistry B $104,10012-10016$

Dresselhaus, M.S., Jorio, A., Souza Filho, A.G., Dresselhaus, G., Saito, R., 2002. Raman spectroscopy on one isolated nanotube. Physica B 323, 15-20. 
Franken, N.A.P., Rodermond, H.M., Stap, F., Haveman, J., van Bree, C., 2006. Clonogenic assay of cells in vitro. Nature Protocols 1, 2315-2319.

Hedderman, T.G., Keogh, S.M., Chambers, G., Byrne, H.J., 2004. Solubilisation of Single walled carbon nanotubes with organic dye molecules. Journal of Physical Chemistry B 108 (49), 18860-18865.

Hedderman, T.G., Keogh, S.M., Chambers, G., Byrne, H.J., 2006. In-depth study into the interaction of single walled carbon nanotubes with anthracene and p-terphenyl. Journal of Physical Chemistry B 110, 3895-3901.

Herzog, E., Casey, A., Lyng, F.M., Chambers, G., Byrne, H.J., Davoren, M., 2007. A new approach to the toxicity testing of carbon based nanomaterials-the clonogenic assay. Toxicology Letters 174, 49-60.

Horáková, K., Švičíková, A., Seemannová, Z., Syrová, D., Bušányová, K., Drobná, Z., Ferenčík, M., 2001. Detection of drug-induced, superoxide-mediated cell damage and its prevention by antioxidants. Free Radical Biology \& Medicine 3, 650-664.

Hurt, R.H., Monthioux, M., Kane, A., 2006. Toxicology of carbon nanomaterials: status, trends, and perspectives on the special issue. Carbon 44, 1028-1033.

Jorio, A., Saito, R., Hafner, J.H., Lieber, C.M., Hunter, M., McClure, T., Dresselhaus, G., Dresselhaus, M.S., 2001. Structural $(\mathrm{n}, \mathrm{m})$ determination of isolated single-wall carbon nanotubes by resonant Raman scattering. Physical Review Letters 86, $1118-1121$.

Jorio, A. Filho, A.G.S., Dresselhaus, G., Dresselhaus, M.S., Swan, A.K., Unlu, M.S. Goldberg, B.B., Pimenta, M.A., Hafner, J.H., Lieber, C.M., Saito, R., 2002. G-band resonant Raman study of 62 isolated single-wall carbon nanotubes. Physical Review B 65, 155412-1-155412-9.

Kataura, H., Kumazawa, Y., Maniwa, Y., Umezu, I., Suzki, S., Ohtsuka, Y., Achiba, Y., 1999. Optical properties of single-wall carbon nanotubes. Synthetic Metals 103, 2555-2558.

Keogh, S.M., Hedderman, T.G. Gregan, E., Farrell, G.F., Chambers, G., Byrne, H.J., 2004. Spectroscopic analysis of single walled carbon nanotube and semiconjugated polymer composites. Journal of Physical Chemistry B 108 (20), 62336241.

Keogh, S.M., Hedderman, T.G., Ruther, M.G., Lyng, F.M., Gregan, E., Farrell, G.F., Byrne, H.J., 2005. Temperature induced nucleation of poly(m-phenylenevinyleneco-2,5-dioctoxy-p-phenylenevinylene) crystallization by HiPco single-walled carbon nanotubes. Journal of Physical Chemistry B 109 (12), 5600-5607.

Kukovecz, A., Kramberger, C., Georgakilas, V., Prato, M., Kuzmany, H., 2002. A detailed Raman study on thin single-wall carbon nanotubes prepared by the HiPco process. European Physical Journal B 28, 223-230.

Kuzmany, H., Plank, W., Hulman, M., Kramberger, Ch., Gruneis, A., Pichler, T.H., Peterlik, H., Kataura, H., Achiba, Y., 2001. Determination of SWCNT diameters from the
Raman response of the radial breathing mode. European Physical Journal B 32, 307-320.

Lima, P.D.L., Vasconcellos, M.C., Montenegro, R.A., Sombra, C.L.M., Bahia, M.O., CostaLotufo, L.V., Pessoa, C.O., Moraes, M.O., Burbano, R.R., 2008. Genotoxic and cytotoxic effects of iron sulfate in cultured human lymphocytes treated in different phases of cell cycle. Toxicology in Vitro 22, 1032-1037.

Monteiro-Riviere, N.A., Inman, A.O., 2006. Challenges for assessing carbon nanomaterial toxicity to the skin. Carbon 44, 1070-1078.

Moulton, S.E., Minett, A.I., Murphy, R., Ryan, K.P., McCarthy, D., Coleman, J.N., Blau, W.J., Wallace, G.G., 2005. Biomolecules as selective dispersants for carbon nanotubes. Carbon 43, 1879-1884.

Ozturk, S., Kaseko, G., Mahaworasilpa, A.T., Coster, H.G.L., 2003. Adaption of cell lines to serum-free culture medium. Hybridoma Hybridomics 22, 267-272.

Pimenta, M.A., Jorio, A., Brown, S.D.M., Filho, A.G.S., Dresselhaus, G., Hafner, J.H. Lieber, C.M., Saito, R., Dresselhaus, M.S., 2001. Diameter dependence of the Raman D-band in isolated single-wall carbon nanotubes. Physical Review B 64 041401-1-041401-4.

Posadaz, A., Sanchez, E., Gutierrez, M.I., Calderon, M., Bertolotti, S., Biasutti, M.A. Garcia, N.A., 2000. Riboflavin and rose Bengal sensitised photo-oxidation of sulfathiazole and succinylsulfathiazole kinetic study and microbiological implications. Dyes and Pigments 45, 219-228.

Puck, T.T., Markus, P.I., 1956. Action of X-rays on mammalian cells. The Journal of Experimental Medicine 103, 653-666.

Rober, P.R., Drewinko, B., 1976. Comparison of in vitro methods to determine druginduced cell lethality. Cancer Research 36, 2182-2188.

Salvador-Morales, C., Flahaut, E., Sim, E., Sloan, J., Green, M.L.H., Sim, R.B., 2006 Complement activation and protein adsorption by carbon nanotubes. Molecular Immunology 43, 193-201.

Spadinger, I., Marples, B., Matthews, J., Skov, K., 1994. Can colony size be used to detect low-dose effects? Radiation Research 138, 21-24.

Wörle-Knirsch, J.M., Pulskamp, K., Krug, H.F., 2006. Oops they did it again! Carbon nanotubes hoax scientists in viability assays. Nanoletters 6, 1261-1268.

Yu, Z., Brus, J., 2001. Rayleigh and Raman Scattering from individual carbon nanotube bundles. Journal of Physical Chemistry B 105, 1123-1134.

Zenin, V.V., Nikolskii, N.N., Skopicheva, V.I., Sorokin, A.B., 1982. Dependence of 3T6 cell proliferation on the serum concentration in the medium. Tsitologiia 24 947-953 (in Russian).

Zirak, P., Penzkofer, A., Schiereis, T., Hegemann, P., Jung, A., Schlichting, I., 2005. Absorption and fluorescence spectroscopic characterisation of BLUF domain of AppA from Rhodobacter sphaeroides. Chemical Physics 315, 142-154. 